Selma O. Algra

Antonius N. J. Schouten

Nicolaas J. G. Jansen

Wim van Oeveren

Felix Haas

Floris Groenendaal

Petra M. A. Lemmers

Ingrid C. van Haastert

Mona C. Toet

Linda S. de Vries

\section{Perioperative and bedside cerebral monitoring identifies cerebral injury after surgical correction of congenital aortic arch obstruction}

Accepted: 3 July 2015

Published online: 28 July 2015

(C) Springer-Verlag Berlin Heidelberg and ESICM 2015

Electronic supplementary material

The online version of this article

(doi:10.1007/s00134-015-3996-6) contains supplementary material, which is available to authorized users.

Dear Editor,

It is becoming increasingly clear that although life-saving, infant cardiac surgery encompasses a high risk of brain injury. Perioperatively performed MRI scans show the injury in most detail (in 40-70 \% of complex cardiac cases), most commonly in the form of white matter injury [1]. In later childhood patients have an increased risk of delay in neurocognitive and motor development [2].

In our cohort of neonates undergoing aortic arch reconstruction while on cardiopulmonary bypass and cooling to deep hypothermia $(n=37)$, we assessed brain injury pre-, intra- and postoperatively using multiple modalities: MRI scans acquired before and after surgery, and perioperative cerebral monitoring using near-infrared spectroscopy (NIRS), amplitude-integrated encephalography (aEEG) and serumbased biomarkers s100b and NSE (for details, see Electronic Supplemental Material). As recently published, we found new postoperative MRI injury in $55 \%$ of patients, and at 2 years of age $(n=32)$, three patients had cerebral palsy, one had a mild motor delay (total motor delay of $13 \%$ ) and two had a cognitive delay (6\%) [3].

As MRI is not always feasible in critically ill patients, we sought to find a bedside marker of cerebral injury for direct use in the paediatric intensive care unit and found that the presence of aEEG seizure activity postoperatively correlated with new MRI injury; thus, 10 of 11 patients with aEEG seizures had a new injury, compared to 9 of 24 patients with a normal aEEG ( $p<0.01$, see Fig. 1a). Also, when assessing the relationship with neurodevelopmental outcome, there was a significant relationship between these aEEG abnormalities and a worse motor outcome (median Psychomotor Developmental Index (PDI) 105 vs. $100, p=0.03$ ), but not cognitive outcome (median Mental Developmental Index (MDI) 100 vs. $100, p=0.10$; see Fig. 1b).

When assessing NIRS measurements, we could not find any correlation to MRI or neurodevelopmental outcome. NSE and s100b did show a relationship with respectively worse motor and cognitive outcome when measured at $4 \mathrm{~h}$ after surgery ( $p<0.01$ and $p=0.04$; for details of statistical analyses, see Electronic Supplemental Material).

The results are in line with those previously published by Clancy et al., who also saw a correlation between conventional EEG and MRI injury in a heterogeneous group of infants undergoing cardiac surgery [4]. In contrast, Gunn et al. did not find a
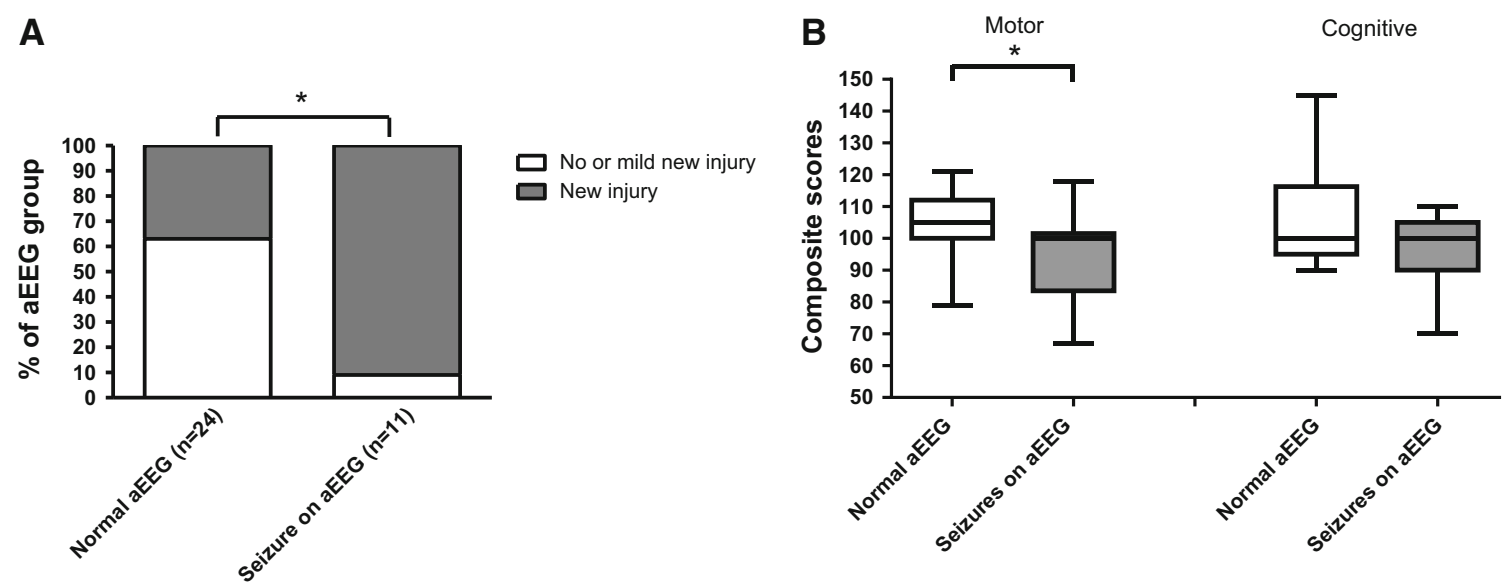

Fig. 1 a Of all cases with electrographic seizures on postoperative aEEG, 10/11(91\%) had a new injury on MRI, whereas this occurred in $9 / 24(37 \%)$ of cases with a normal aEEG $(p<0.01)$.

b Electrographic seizures on postoperative aEEG are significantly related to a lower motor outcome $(p=0.03)$ but not cognitive outcome $(p=0.10)$ 
relationship between seizures and outcome but did see that a slower recovery to a normal aEEG pattern after surgery was associated with a worse outcome [5]. We could not confirm the latter. Of note, Gunn et al. reported more intraoperative than postoperative seizures, whereas we saw more artefacts due to ECG and cardiopulmonary bypass than convincing seizure activity.

In conclusion, in patients undergoing surgical correction of congenital aortic arch obstruction, postoperative aEEG cerebral monitoring proved effective in identifying patients at risk of cerebral injury. In addition, s100b and NSE are promising biomarkers, associated with early neurodevelopmental outcome, whereas NIRS was not related to cerebral injury.

\section{Compliance with ethical standards}

Conflicts of interest The authors declare no conflicts of interest.

\section{References}

1. Creighton DE, Robertson CM, Sauve RS et al (2007) Neurocognitive, functional, and health outcomes at 5 years of age for children after complex cardiac surgery at 6 weeks of age or younger. Pediatrics. doi:10.1542/peds.2006-3250
2. Rollins CK, Newburger JW (2014) Cardiology patient page. Neurodevelopmental outcomes in congenital heart disease. Circulation. doi: $10.1161 /$

CIRCULATIONAHA.114.008556

3. Algra SO, Jansen N, van der Tweel I et al (2013) Neurological Injury after neonatal cardiac surgery: a randomized controlled trial of two perfusion techniques. Circulation. doi:

10.1161/

CIRCULATIONAHA.113.003312

4. Clancy RR, Sharif U, Ichord R et al (2005) Electrographic neonatal seizures after infant heart surgery. Epilepsia. doi: 10.1111/j.0013-9580.2005.22504.x

5. Gunn JK, Beca J, Hunt RW, Olischar M, Shekerdemian LS (2012) Perioperative amplitude-integrated EEG and neurodevelopment in infants with congenital heart disease. Intensive Care Med. doi:10.1007/s00134-012-2608-y

S. O. Algra (-) · F. Haas Department of Pediatric Cardiothoracic Surgery, University Medical Center Utrecht, KG.01.319.0, UMC Utrecht, Lundlaan 6, 3584 Utrecht, The Netherlands e-mail: selmaalgra@hotmail.com Tel.: 0031-887554002
A. N. J. Schouten

Department of Anesthesiology and Intensive Care and Emergency Medicine, University Medical Center Utrecht, Groningen, The Netherlands

S. O. Algra - N. J. G. Jansen

Department of Pediatric Intensive Care, University Medical Center Utrecht, Groningen, The Netherlands e-mail: n.j.g.jansen@umcutrecht.nl

F. Groenendaal - P. M. A. Lemmers . I. C. van Haastert - M. C. Toet .

L. S. de Vries

Department of Neonatology, University Medical Center Utrecht, Groningen, The Netherlands

W. van Oeveren

HaemoScan, Groningen,

The Netherlands 